ARTICLE OPEN

\title{
Precursor of pair-density wave in doping Kitaev spin liquid on the honeycomb lattice
}

\author{
Cheng Peng ${ }^{1}$, Yi-Fan Jiang ${ }^{1}$, Thomas P. Devereaux $\mathbb{D}^{1,2}$ and Hong-Chen Jiang $\mathbb{1}^{1 凶}$
}

We study the effects of doping the Kitaev model on the honeycomb lattice where the spins interact via the bond-directional interaction $J_{K}$, which is known to have a quantum spin liquid as its exact ground state. The effect of hole doping is studied within the $t-J_{K}$ model on a three-leg cylinder using density-matrix renormalization group. Upon light doping, we find that the ground state of the system has a dominant quasi-long-range charge-density-wave correlations but short-range single-particle correlations. In the pairing channel, the even-parity superconducting correlation is dominant with $d$-wave-like symmetry, which oscillates in sign as a function of separation with a period equal to that of the spin-density wave and two times the charge-density wave. Although these correlations fall rapidly (possibly exponentially) at long distances, this is never-the-less the example where a pair-density wave is the leading instability in the pairing channel on the honeycomb lattice.

npj Quantum Materials (2021)6:64; https://doi.org/10.1038/s41535-021-00363-0

\section{INTRODUCTION}

The pair-density wave (PDW) is a superconducting (SC) state in which the order parameter varies periodically in space in such a way that its spatial average vanishes ${ }^{1,2}$. The first example of PDW is the Fulde-Ferrell-Larkin-Ovchinnikov (FFLO) state ${ }^{3,4}$ when a Zeeman magnetic field, $H$, is applied to a s-wave superconductor so that the Fermi surface is spin-split. The SC order has a wavevector $Q \sim \mu_{B} H / E_{F}$, which is typically very small. The $L O$ version of this state is accompanied by an induced magnetization density wave and a charge-density wave (CDW) with ordering wavevectors $K=2 Q$. Intense interest in a somewhat different sort of PDW state has emerged due to recent discoveries in underdoped cuprate superconductors, where a direct observation of PDW has been made experimentally via local Cooper pair tunneling and scanning tunneling microscopy in $\mathrm{Bi}_{2} \mathrm{Sr}_{2} \mathrm{CaCu}_{2} \mathrm{O}_{8+x}{ }^{5-7}$, as well as the dynamical inter-layer decoupling observed in $1 / 8$ hole-doped $\mathrm{La}_{2} \mathrm{BaCuO}_{4}{ }^{8,9}$. While similar in having oscillatory SC order and associated $K=2 Q \mathrm{CDW}$ order, this PDW is conjectured to be stable in zero magnetic field (zero net magnetization), have an ordering vector that is independent of $H$ (at least for small or vanishing $H$ ), and moreover can either have no associated magnetic order, or possibly have spin-density wave order (SDW) with the same ordering vector $Q$.

Although much is known about the properties of the PDW state $^{1,2,10}$ there are very few microscopic models, which are shown to have PDW ground states. These include the one-dimensional (1D) Kondo-Heisenberg model with 1D electron gas coupled to a spin chain ${ }^{11}$, the extended two-leg Hubbard-Heisenberg model ${ }^{12}$, and strong coupling limit of the Holstein-Hubbard Model ${ }^{13,14}$. The evidence of PDW is also observed in the $t-J$ model with four-spin ring exchange on a four-leg triangular lattice ${ }^{15}$ and an extended Hubbard model with a staggered spin-dependent magnetic flux per plaquette on a three-leg triangular lattice ${ }^{16}$. However, there is no evidence of PDW ordering found in more standard models even with second neighbor interactions ${ }^{17-20}$.

Theoretically, it has been proposed that superconductivity can also emerge in doping quantum spin liquids (QSLs), which are exotic phases of matter that exhibit a variety of features associated with their topological character ${ }^{21-23}$. The QSLs can be viewed as insulating phases with preexisting electron pairs such that upon light doping they might automatically yield hightemperature superconductivity ${ }^{24-31}$. Indeed, recent numerical studies using density-matrix renormalization group (DMRG) have provided strong evidences that lightly doping the QSL and chiral spin liquid on the triangular lattice will naturally give rise to nematic $d$-wave ${ }^{32}$ and $d \pm i d$-wave superconductivity ${ }^{33}$, respectively. Although dominant SC correlations have been observed in both systems, there is still no evidence for PDW ordering.

In this paper, we define a $t$-J-like extension of the Kitaev model on the honeycomb lattice (Fig. 1) so as to address the question of whether SC emerges upon light doping. The Kitaev model, i.e., $J_{K}$ term in Eq. (4), is exactly solvable and has a gapless spin liquid as its exact ground state ${ }^{34}$. Moreover, it has potential experimental realizations in magnets with strong spin-orbit coupling such as $\mathrm{Na}_{2} \mathrm{IrO}_{3}$ and $\mathrm{a}-\mathrm{RuCl}_{3}{ }^{35-40}$. This provides us a unique theoretical opportunity to test the physics of doping QSLs and may also give us some hints for understanding the mechanism of hightemperature superconductivity in the cuprates. Theoretically, doping Kitaev spin liquid (KSL) has been studied and distinct metallic states were proposed ${ }^{41-45}$. These include the $p$-wave superconductivity ${ }^{41,42}, d$-wave superconductivity ${ }^{43}$, topological superconductivity ${ }^{44}$, and Fermi liquid state ${ }^{45}$. However, controlled results of the sort that can be obtained using density-matrix renormalization group (DMRG) are still lacking concerning the phase(s) that arise upon doping the KSL.

\section{RESULTS}

\section{Principal results}

In the present paper, we study the lightly doped Kitaev model in Eq. (4) on the honeycomb lattice using DMRG ${ }^{46}$. Based on DMRG calculations on three-leg cylinders, we find that upon light-doping the KSL state, the system exhibits power-law CDW correlations at long distances corresponding to a local pattern of partially filled

\footnotetext{
${ }^{1}$ Stanford Institute for Materials and Energy Sciences,SLAC National Accelerator Laboratory and Stanford University, Menlo Park, CA, USA. ${ }^{2}$ Department of Materials Science and Engineering, Stanford University, Stanford, CA, USA. ${ }^{凶}$ email: hcjiang@stanford.edu
} 
charge stripes. For three-leg cylinders, the wavelength of CDW, i.e., the spacings between two adjacent charge strips in the $\mathbf{e}_{1}$ direction, is $\lambda_{c}=a_{0} / 3 \delta$, where $a_{0}$ is the length of unit cell. This corresponds to an ordering wavevector $K=3 \pi \delta / a_{0}$ with two thirds of a doped hole per CDW unit cell.

We find that the even-parity SC correlations are the most pronounced SC correlations, far dominant compared with the odd-parity SC or topological superconductivity ${ }^{41,42,44}$. Moreover, the dominant pairing channel oscillates in sign as a function of distance, which is consistent with the striped PDW ${ }^{2}$. Its wavelength $\lambda_{\mathrm{sc}}=2 a_{0} / 3 \delta$ is the same as that of the SDW correlations $\lambda_{\mathrm{s}}=2 a_{0} / 3 \delta$ and two times of that of the CDW $\lambda_{\mathrm{c}}=$ $a_{0} / 3 \delta$. Correspondingly, the SC ordering wavevector $Q=3 \pi \delta / 2 a_{0}$ is half of the ordering wavevector $K=3 \pi \delta / a_{0}$ of the CDW. However, our numerical results are inconsistent with the theoretical predictions ${ }^{39-43}$ as we find that both the singleparticle Green functions and SC correlations are short-ranged, which decay exponentially at long distances. Although quasi-longrange SC correlations are not seen in the range of parameters we have studied, short-range correlations are fairly strong with the corresponding correlation length $\xi_{\mathrm{sc}} \geq 3 a_{0}$. This is comparable with the cylindrical width and is notably larger than that of doping the

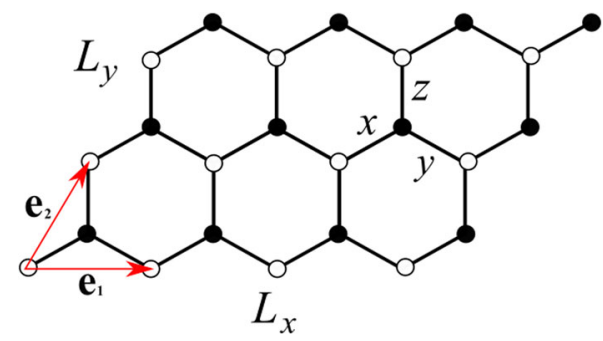

Fig. 1 The schematic three-leg cylinder on the honeycomb lattice. The open (filled) circle denotes $A(B)$ sub-lattice, and $x, y$, and $z$ label the three different bonds. Periodic (open) boundary condition is imposed along the direction specified by the lattice basis vector $\mathbf{e}_{2}\left(\mathbf{e}_{1}\right) . L_{x}\left(L_{y}\right)$ is the number of unit cells in the $\mathbf{e}_{1}\left(\mathbf{e}_{2}\right)$ direction.
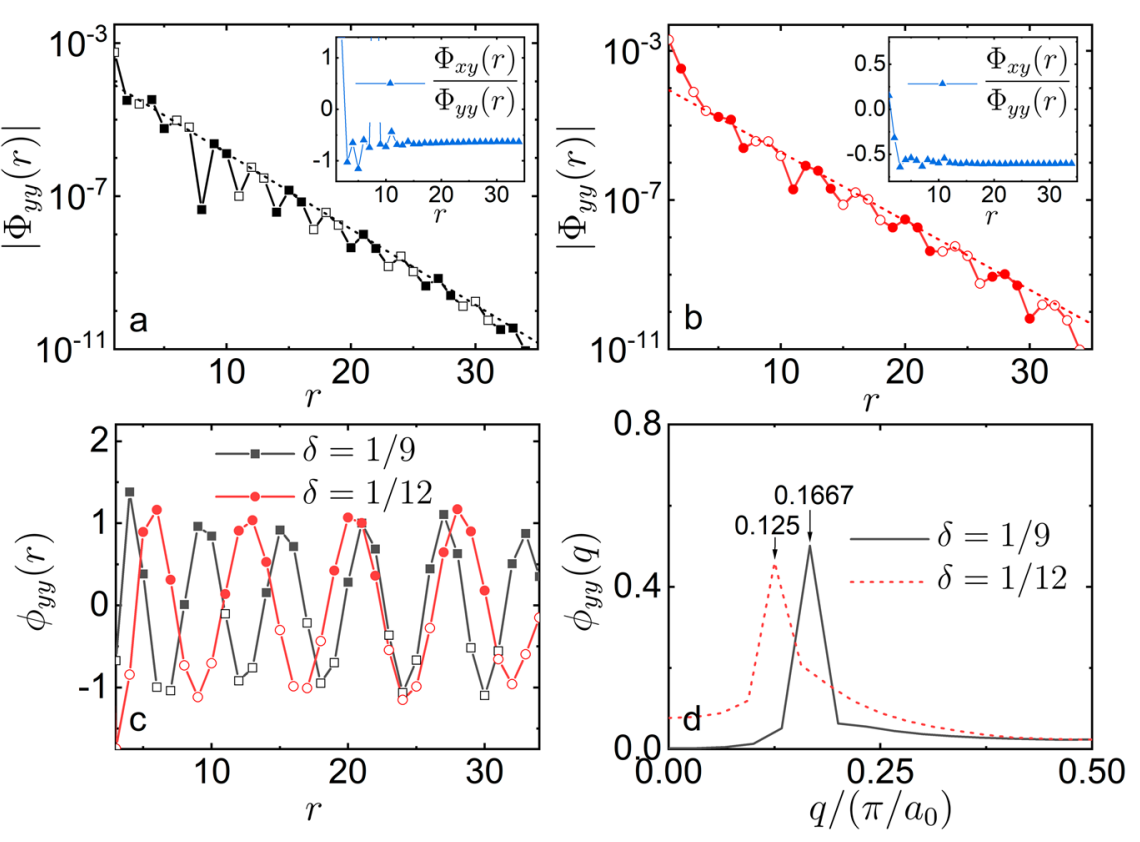

To test the possibility of superconductivity, we have calculated the equal-time SC pair-pair correlations. A diagnostic of the SC order is the SC pair-pair correlation function, defined as

$\Phi_{a \beta}(r)=\left\langle\Delta_{a}^{\dagger}\left(x_{0}, y\right) \Delta_{\beta}\left(x_{0}+r, y\right)\right\rangle$,

where $\Delta_{a}^{\dagger}(x, y)=\frac{1}{\sqrt{2}}\left[\hat{c}_{(x, y), \uparrow}^{\dagger} \hat{c}_{(x, y)+\alpha, \downarrow}^{\dagger}-\hat{c}_{(x, y), \downarrow}^{\dagger} \hat{c}_{(x, y)+a, \uparrow}^{\dagger}\right]$ is the evenparity SC pair-field creation operator, where the bond orientations are labeled as $a=x, y, z$ (Fig. 1). $\left(x_{0}, y\right)$ is the reference bond with $x_{0} \sim \tilde{L}_{x} / 4$ to minimize the boundary effect and $r$ is the distance between two bonds in the $\mathbf{e}_{1}$ direction. We have also calculated the odd-parity SC correlations to test the possibility of $p$-wave or topological superconductivity. However, we find that they are much weaker than the even-parity SC correlations (see Supplementary Discussion for details), suggesting that $p$-wave or topological superconductivity is unlikely. Therefore, we will focus on the even-parity SC correlation in this paper.

Figure 2 shows the SC pair-pair function $\Phi_{y y}(r)$ for doping $\delta=$ $1 / 12$ and $1 / 9$. The SC correlation shows clear spatial oscillation for both doping levels, which can be well fitted by $\Phi_{y y}(r) \sim f(r) * \phi_{y y}(r)$ for a large region of $r$ as we will discuss below. Here, $f(r)$ is the envelope function and $\phi_{y y}(r)$ is a spatial oscillatory function. At long distances, the envelope function $f(r)$ is consistent with an exponential decay, i.e., $f(r) \sim \mathrm{e}^{-r / \xi_{s c}}$, as shown in Fig. $2 \mathrm{a}$, b. The extracted correlation length is $\xi_{s c} \geq 3 a_{0}$, which is comparable with the cylindrical width $L_{y}=3 a_{0}$. Alternatively, the SC correlation at long distances can also be fitted by a power law (see Supplementary Discussion for details), i.e., $f(r) \sim r^{-K_{s c}}$, with an exponent $K_{\mathrm{sc}}>4$ for both doping levels $\delta=1 / 12$ and $1 / 9$. We have also measured other types of SC correlations $\Phi_{a \beta}(r)$, which are provided in the Supplementary Discussion. While they are slightly weaker than $\Phi_{y y}(r)$ due to the broken symmetry induced by the cylindrical geometry, they have very similar decaying behavior

Fig. 2 Superconducting correlations and their spacial oscillation. Superconducting correlations $\left|\Phi_{y y}(r)\right|$ on three-leg cylinder at doping level a $\delta=1 / 9$ and $\mathbf{b} \delta=1 / 12$, where dashed lines denote fittings to an exponential function $f(r) \sim \mathrm{e}^{-r / \xi_{s c}}$. Insets are the ratio $\Phi_{x y}(r) / \Phi_{y y}(r)<0$, where the out of phase indicates $d$-wave type pairing. c The normalized function $\phi_{y y}(r)=\Phi_{y y}(r) / f(r)$ at $\delta=1 / 9$ and $\delta=1 / 12$, which directly reflects the spatial oscillation of $\Phi_{y y}(r)$. d Fourier transformation $\phi_{y y}(q)$ of $\phi_{y y}(r)$ at $\delta=1 / 12$ and $\delta=1 / 9$, where peaks lie at $Q=3 \pi \delta / 2 a_{0}$ giving the total momentum of the pairing. Note that filled (open) symbols denote positive (negative) value. 

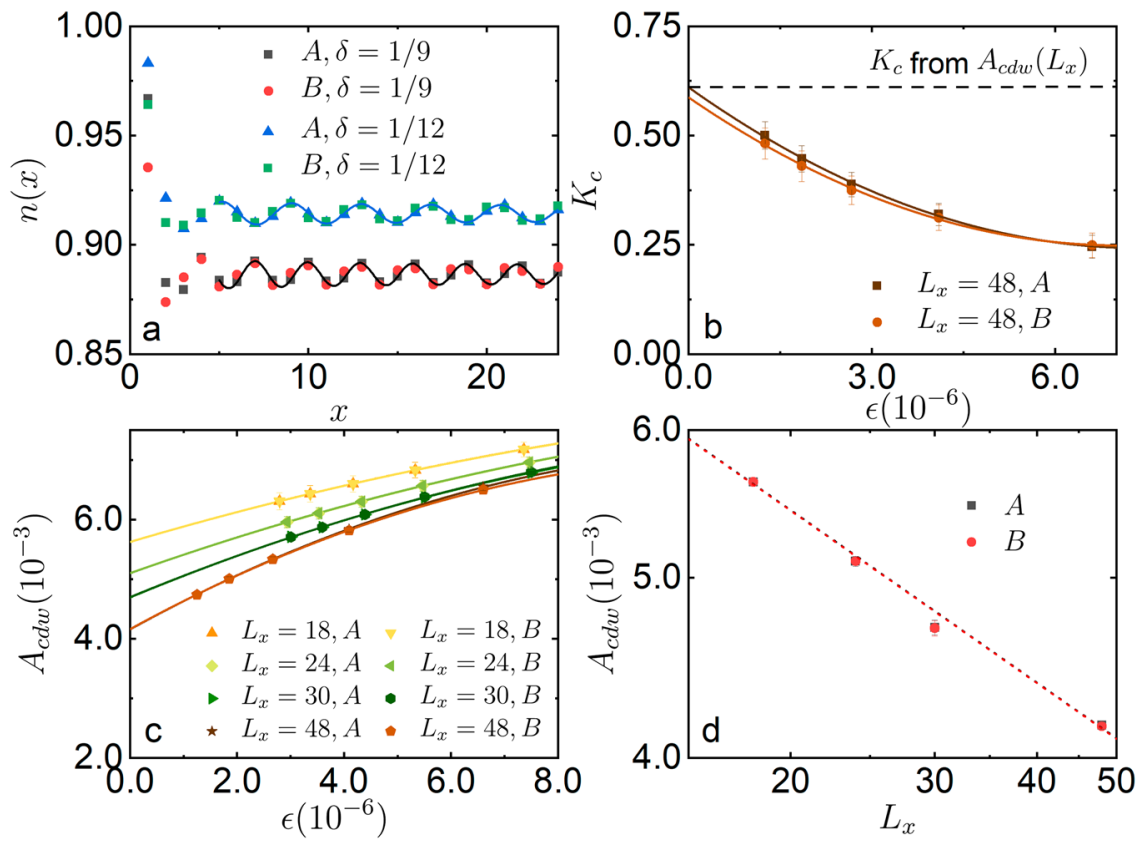

Fig. 3 CDW and Luttinger exponent. a Charge-density profiles $n(x)$ for three-leg cylinder of length $L_{x}=48$ at doping levels $\delta=1 / 9$ and $\delta=1 /$ 12 on $A$ and $B$ sub-lattices, respectively. The solid lines denote the fitting using Eq. (2). $\mathbf{b}$ The extracted exponent $K_{c}$ at $\delta=1 / 9$ as a function of truncation error $\epsilon$. The dashed line denotes $K_{c}$ extracted from $A_{c d w}\left(L_{x}\right)$ in $(\mathbf{d})$. c Convergence and length dependence of the CDW amplitude $A_{c d w}$ for three-leg cylinder at $\delta=1 / 9$ as a function of truncation error $\epsilon$. The solid lines denote fitting using second-order polynomial function. d Finite-size scaling of $A_{\mathrm{cdw}}\left(L_{x}\right)$ as a function of $L_{x}$ in a double-logarithmic plot at $\delta=1 / 9$, where the dashed line denotes the fitting to a power law $A_{\mathrm{cdw}}\left(L_{x}\right) \sim L_{x}^{-K_{\mathrm{c}} / 2}$.

with $\Phi_{y y}(r)$. Although the SC correlations can be fitted by either functions, it is clear that the SC susceptibility will not diverge in the thermodynamic limit.

The spatial oscillation of the SC correlation $\Phi_{y y}(r)$ is characterized by the normalized correlation $\phi_{y y}(r)=\Phi_{y y}(r) / f(r)$, as shown in Fig. 2c. It is clear that $\phi_{y y}(r)$ varies periodically in real space and can be well fitted by $\phi_{y y}(r) \sim \sin (Q r+\theta)$ for both cases. This is consistent with the PDW state with vanishing spatial average of $\phi_{y y}(r)$. The ordering wavevector of the PDW is $Q=3 \pi \delta / 2 a_{0}$ as indicated by the peak position of the Fourier transformation $\phi_{y y}(q)$ of $\phi_{y y}(r)$ (Fig. 2d) and $\theta$ is a fitting phase factor. The corresponding wavelength is $\lambda_{\mathrm{sc}}=2 a_{0} / 3 \delta$, which is $\lambda_{\mathrm{sc}}=8 a_{0}$ for $\delta=1 / 12$ and $\lambda_{\mathrm{sc}}=6 a_{0}$ for $\delta=1 / 9$. As we will see below that the relation $\lambda_{\mathrm{sc}}=\lambda_{\mathrm{s}}$ $=2 \lambda_{c}$ can be clearly seen in our results as expected from that of striped PDW. Here, $\lambda_{c}$ and $\lambda_{s}$ are the wavelengths of the CDW and SDW, respectively. According to Ginzburg-Landau theory, the development of $K=2 Q$ charge oscillation corresponds to the cubic term $\rho_{K} \Delta_{Q}^{*} \Delta_{-Q}$ in the free energy, where $\rho_{K}$ and $\Delta_{Q}$ are the charge density and PDW order parameters with corresponding momenta $K$ and $Q$, respectively. This is distinct from the CDW modulated superconductivity with term $\rho_{Q} \Delta_{0}^{*} \Delta_{-Q}$ with coexisting dominant uniform $\Delta_{0}$ and secondary stripe pairing $\Delta_{Q}$.

To identify the pairing symmetry, we have calculated the SC correlations using both real-valued and complex-valued DMRG simulations. We first rule out the $d \pm i d$-wave symmetry as we find that both the wavefunction and SC correlations are real while their imaginary parts are zero. We have further analyzed the relative phase of different SC correlations, e.g., $\Phi_{x y}(r) / \Phi_{y y}(r)$ in the insets of Fig. $2 a, b$, where a clear out of phase can be observed as $\Phi_{x y}(r) /$ $\Phi_{y y}(r)<0$. Therefore, we conclude that the pairing symmetry of the PDW is consistent with $d$-wave.

\section{Charge-density wave}

In addition to SC correlations, we have also measured the chargedensity profiles to describe the charge-density properties of the system. The rung charge density $n(x)=\sum_{y=1}^{L_{y}} n(x, y) / L_{y}$ is shown

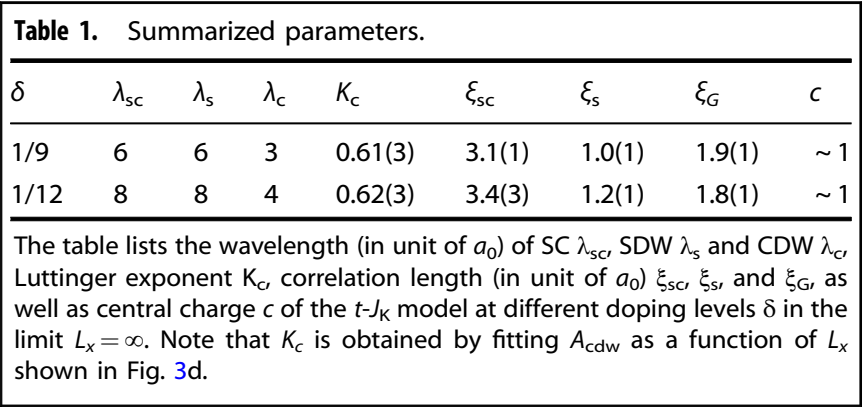

in Fig. 3, where $x$ is the rung index of the cylinder and $n(x, y)$ is the local charge-density on site $i=(x, y)$. It is clear that the charge density varies periodically in real space along the $\mathbf{e}_{1}$ direction with the wavelength $\lambda_{c}=a_{0} / 3 \delta$, i.e., $\lambda_{c}=4 a_{0}$ and $\lambda_{c}=3 a_{0}$ for $\delta=1 / 12$ and $\delta=1 / 9$, respectively. Therefore, there are two thirds of a doped hole in each CDW unit cell. Moreover, it is clear that the relation $\lambda_{\mathrm{sc}}=2 \lambda_{\mathrm{c}}$ holds for both $\delta=1 / 12$ and $\delta=1 / 9$, which is consistent the striped PDW state. Different with SC correlations, we find power-law decay of the charge-density correlation at long distances. The Luttinger exponent $K_{\mathrm{c}}$ of the power-law decay can be extracted by fitting the charge-density oscillation (Friedel oscillation) induced by the open boundaries of the cylinder ${ }^{48,49}$

$n(x)=n_{0}+\delta n * \cos (K x+\theta) x^{-K_{c} / 2}$.

Here, $x$ is the distance in the $\mathbf{e}_{1}$ direction from the open boundary, $n_{0}$ the average density and $K$ is the ordering wavevector. $\delta n$ and $\theta$ are the model-dependent constants. Examples of the fitting using Eq. (2) on the a sub-lattice are given in Fig. 3a for both doping $\delta=$ $1 / 12$ and $\delta=1 / 9$, where four data points near the boundary are removed to minimize boundary effect for a more reliable fit. The extracted exponent $K_{c}$ is given in Table 1 . It is clear that $K_{c}<1$, 

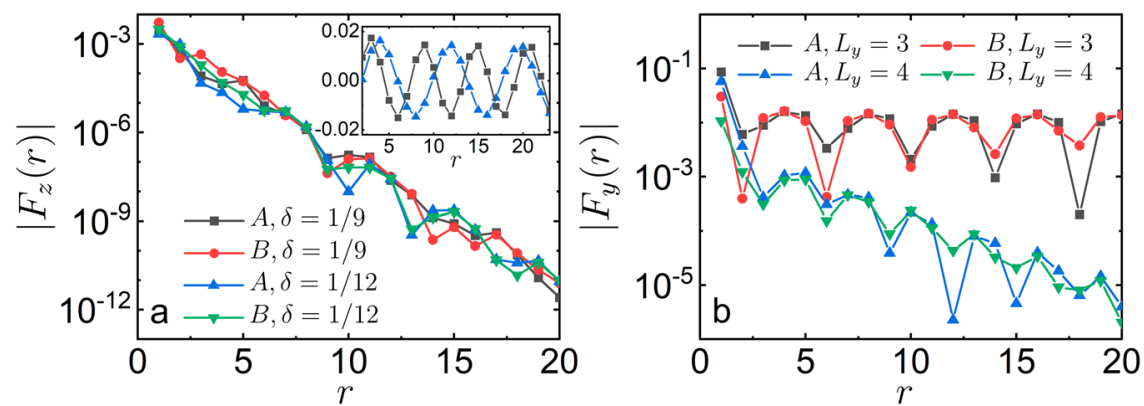

Fig. 4 Spin-spin correlations. a $F_{z}(r)$ for three-leg cylinder at different doping levels $\delta$ and $\mathbf{b} F_{y}(r)$ for both three and four-leg cylinders at doping level $\delta=1 / 12$. Inset shows the SDW spatial oscillation, i.e., $F_{y}(r)$, with the black square and blue triangle symbols are for $\delta=1 / 9$ and $\delta=1 / 12$, respectively.
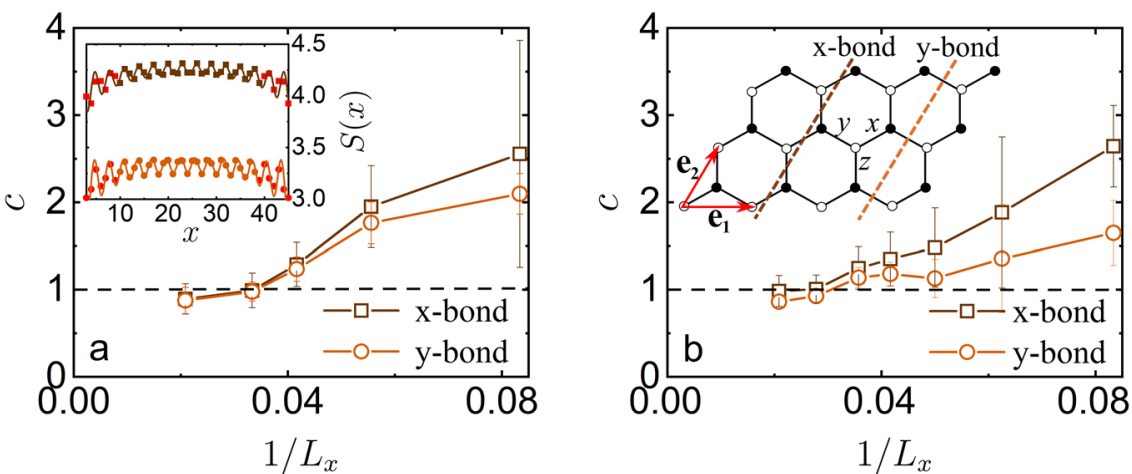

Fig. 5 Central charge. The extracted central charge $c$ as a function of $L_{x}$ using Eq. (3) for three-leg cylinder at doping level a $\delta=1 / 9$ and $\mathbf{b} \delta=$ 1/12. Error bars denote numerical uncertainty. Inset in a is the entanglement entropy $S(x)$ on three-leg cylinder of length $L_{x}=48$, a few data points in red are removed to minimize the boundary effect. Inset in $\mathbf{b}$ shows the smooth boundaries parallel to the $\mathbf{e}_{2}$ direction through $x$ and $y$-bond labeled by the dashed lines.

which demonstrates the dominance of the charge-density correlations.

Alternatively, we can estimate $K_{\mathrm{c}}$ from the amplitude $A_{\mathrm{cdw}}\left(L_{x}\right)$ of the charge-density modulation ${ }^{19}$. For a given cylinder of length $L_{x}$, the CDW amplitude $A_{\mathrm{cdw}}\left(L_{x}\right)$ can be obtained by fitting the centralhalf region of the charge-density profile $n(x)^{18-20}$. For quasi-longrange charge order, the amplitude should follow $A_{c d w}\left(L_{x}\right) \propto L_{x}^{-K_{c} / 2}$ with similar $K_{c}$ with that obtained from Friedel oscillation in Eq. (2). This is indeed the case as shown in Fig. 3c, d, where the exponent $K_{c}$ is given in Fig. $3 b$ and Table 1.

\section{Spin-spin and single-particle correlations}

To describe the magnetic properties of the system, we have further calculated the spin-spin correlation function $F_{\gamma}(r)=\left\langle S_{i_{0}}^{\gamma} S_{i_{0}+r}^{\gamma}\right\rangle$. Here, $r$ is the distance between two sites in the $\mathbf{e}_{1}$ direction and $\gamma=x, y, z . i_{0}=\left(x_{0}, y\right)$ is the reference site with $x_{0} \sim \tilde{L}_{x} / 4$. For the pure Kitaev model without doping, it is known that $F_{\gamma}(r)$ is nonzero only for NN sites ${ }^{34,50}$. Upon doping, the $Z_{2}$ flux on each hexagonal plaquette is no longer a conserved quantity, and the spin-spin correlation functions become nonzero even at long distance. For both doping levels $\delta=1 / 12$ and $\delta=1 / 9$ on three-leg cylinders, we find that both $F_{x}(r)$ and $F_{z}(r)$ decay exponentially as $F(r) \sim \mathrm{e}^{-r / \xi_{\mathrm{s}}}$, where the spin-spin correlation length is given in Table. 1. On the contrary, $F_{y}(r)$ appears to be long-range ordered as shown in Fig. 4b. This is unexpected but allowed theoretically since there is no continuous spin symmetry in the system. Moreover, we find that the spin-spin correlation $F_{y}(r)$ shows clear spatial oscillation as shown in the inset of Fig. 4a with a wavelength $\lambda_{s}$ that is the same as that of the SC correlation (see Table 1), i.e., $\lambda_{s}=\lambda_{s c}$, which is consistent with the striped PDW. However, our results suggest that the long-range correlation $F_{y}(r)$ is special to three-leg cylinder. On the contrary, $F_{y}(r)$ decays exponentially on the wider four-leg cylinders (Fig. 4b), which is similar with both $F_{x}(r)$ and $F_{z}(r)$. However, the evidences of the striped PDW are robust for both three and four-leg cylinders with similar sign-changing SC correlations (see Supplementary Discussion for details).

In addition to superconductivity, we have also measured the single-particle Green function $G_{\sigma}(r)=\left\langle c_{i}^{\dagger} c_{i+r}\right\rangle$ to test the possibility of Fermi liquid state ${ }^{45}$. It is clear that the single-particle Green function decays exponentially at long distances as $G_{\sigma}(r) \sim \mathrm{e}^{-r / \xi_{G}}$ (see Supplementary Discussion for details). Here, $\xi_{\mathrm{G}}$ is the correlation length, which is summarized in Table 1. As a result, our study suggests that the Fermi liquid state is unlikely in the lightly hole-doped KSL.

\section{Central charge}

Our results suggest that the ground state of the lightly hole-doped KSL has quasi-long-range CDW correlation with gapless charge mode. To show this, we have calculated the von Neumann entanglement entropy $S(x)=-\operatorname{Tr}\left[\rho_{x} \ln \rho_{x}\right]$, where $\rho_{x}$ is the reduced density matrix of subsystem with length $x$. For finite 1D critical system of length $L_{x}$ with open boundaries, the central charge $c$ or the number of gapless mode can be obtained using $^{51,52}$,

$$
\begin{aligned}
S(x)= & \frac{c}{6} \ln \left[\frac{4\left(L_{x}+1\right)}{\pi} \sin \frac{\pi(2 x+1)}{2\left(L_{x}+1\right)}\left|\sin k_{F}\right|\right] \\
& +\frac{a \pi \sin \left[k_{F}(2 x+1)\right]}{4\left(L_{x}+1\right) \sin \frac{\pi(2 x+1)}{2\left(L_{x}+1\right)}\left|\sin k_{F}\right|}+\tilde{C} .
\end{aligned}
$$

Here, $k_{\mathrm{F}}$ denotes the Fermi momentum, $a$ and $\tilde{c}$ are modeldependent parameters. Figure 5a shows an example of $S(x)$ for three-leg cylinder at doping level $\delta=1 / 9$. Here we have calculated $S(x)$ by dividing the system into two parts with smooth boundary ${ }^{53}$ through both $x$ and $y$ bonds as shown in the inset of Fig. $5 b$. 
The extracted central charge $c$ is given in Fig. 5 as a function of $L_{x}$ at doping levels $\delta=1 / 9$ and $1 / 12$. It is clear that the central charge quickly converges to $c=1$ with the increase of $L_{x}$, although it deviates notably from $c=1$ on short cylinders due to finite-size effect. Therefore, our results show that there is one gapless charge mode that is consistent with the quasi-long-range CDW correlations. We have obtained similar results on four-leg cylinders, which are provided in the Supplementary Discussion.

\section{DISCUSSION}

Admittedly, the DMRG calculations are carried out on finite length cylinders. However, based on the results we have obtained we conjecture that the exact ground state for an infinite long threeleg cylinder has the following properties: (1) There is a single gapless charge mode and a gap (which produces exponentially falling correlations) for all spin carrying excitations. (2) Long-range SDW order with the ordered moment oriented in the $y$ direction that is along the circumference of the cylinder-and an ordering vector $Q=3 \pi \delta / 2$; the connected spin correlations fall exponentially with a correlation length $\xi_{\mathrm{s}} \sim a_{0}$. (3) There are power-law CDW correlations with an exponent $K_{c} \sim 2 / 3$ and an ordering vector $K=2 Q$. (4) There are strong even-parity d-wave-like PDW correlations with an ordering vector $Q$, which fall either exponentially with a correlation length $\xi_{\mathrm{sc}} \sim 3 a_{0}$ or possibly with a high power-law $K_{\mathrm{sc}} \geq 4$. 5) All other forms of SC correlationsthose corresponding to odd-parity pairing or spatially uniform even-parity pairing - are much weaker in comparison with the PDW correlations.

There are many aspects of these observations that are surprising, and will need to be understood theoretically. The PDW correlations are sufficiently short-ranged that one would infer (based on any reasonable conjecture concerning their time dependence) that the corresponding PDW susceptibility would be finite. Thus, at present, we can only conclude that the present results are suggestive of a possible PDW ordered state for the 2D (infinite leg) version of this model. However, it is notable that the favored forms of order are remarkably reminiscent of those conjectured to be present in the cuprate hightemperature superconductor, LBCO, where direct evidence exists of stripe SDW order with wavevector $Q \approx 2 \pi \delta$ and CDW order with ordering vector $K=2 Q$, and indirect evidence has been adduced for PDW order with ordering vector $Q$.

In this paper, we primarily focus on the lightly doped Kitaev model, it will be interesting to study the higher doping case as well as the extend Kitaev model with further neighbor hopping, which is shown to be essential to enhance the superconductivity on the square lattice ${ }^{19,20}$. As other terms such as the Heisenberg interaction and spin-orbit couplings are also present in real materials such as $\mathrm{Na}_{2} \mid \mathrm{rO}_{3}$ and $a-\mathrm{RuCl}_{3}{ }^{35-40}$, it will be interesting to study these systems as well.

\section{METHODS}

\section{Model Hamiltonian}

We employ DMRG ${ }^{46}$ to investigate the ground state properties of the holedoped Kitaev model on the honeycomb lattice defined by the Hamiltonian

$H=-t \sum_{\langle j\rangle, \sigma}\left(c_{i \sigma}^{\dagger} c_{j \sigma}+\right.$ h.c. $)+J_{K} \sum_{\langle i j\rangle} S_{i}^{\gamma} S_{j}^{\gamma}$.

Here, $c_{i \sigma}^{\dagger}\left(c_{i \sigma}\right)$ is the electron creation (annihilation) operator with spin- $\sigma$ on site $i=\left(x_{i}, y_{i}\right), S_{i}^{\gamma}$ is the $\gamma$-component of the $S=1 / 2$ spin operator on site $i$, where $y=x, y, z$ labels the three different links of the hexagonal lattice as illustrated in Fig. 1. $\langle i j\rangle$ denotes nearest-neighbor (NN) sites and the Hilbert space is constrained by the no-double occupancy condition, $n_{i} \leq 1$, where $n_{i}=\sum_{\sigma} c_{i \sigma}^{\dagger} c_{i \sigma}$ is the electron number operator. At half-filling, i.e., $n_{i}=1$, Eq. (4) reduces to the Kitaev model, which is known to have a gapless spin liquid ground state that can be gapped out into a non-Abelian topological phase by certain time-reversal symmetry perturbations ${ }^{34,54}$.

\section{Numerical details}

The lattice geometry used in our simulations is depicted in Fig. 1, where $\mathbf{e}_{1}=(\sqrt{3}, 0)$ and $\mathbf{e}_{2}=(\sqrt{3} / 2,3 / 2)$ denote the two basis vectors. We consider honeycomb cylinders with periodic and open boundary conditions in the $\mathbf{e}_{2}$ and $\mathbf{e}_{1}$ directions, respectively. We focus on cylinders of width $L_{y}$ and length $L_{x}$ where $L_{y}$ and $L_{x}$ are the number of unit cells ( $L_{y}$ and $\tilde{L}_{x}=2 L_{x}$ are the number of sites) along the $\mathbf{e}_{2}$ and $\mathbf{e}_{1}$ directions, respectively. The total number of sites is $N=2 \times L_{y} \times L_{x}$. The hole doping concentration is defined as $\delta=N_{\mathrm{h}} / N$, where $N_{\mathrm{h}}$ is the number of doped holes. We set $J_{K}=1$ as an energy unit and consider $t=3$. In this paper, we focus primarily on three-leg cylinders, i.e., $L_{y}=3 a_{0}$, of length $L_{x}=12 a_{0} \sim$ $48 a_{0}$ (i.e., $\tilde{L}_{x}=24 \sim 96$ ), at doping levels $\delta=1 / 12$ and $1 / 9$. We keep up to $m=8000$ number of states in each DMRG block with a typical truncation error $\epsilon \sim 10^{-6}$. We find similar results on four-leg cylinders. Further details of the numerical simulation are provided in the Supplementary Discussion.

\section{DATA AVAILABILITY}

The authors declare that the main data supporting the findings of this study are available within the article and its Supplementary Discussion. Extra data are available from the corresponding author upon reasonable request.

\section{CODE AVAILABILITY}

The codes implementing the calculations of this study are available from the corresponding author upon request.

Received: 8 February 2021; Accepted: 8 June 2021;

Published online: 02 July 2021

\section{REFERENCES}

1. Berg, E., Fradkin, E., Kivelson, S. A. \& Tranquada, J. M. Striped superconductors: how spin, charge and superconducting orders intertwine in the cuprates. New J. Phys. 11, 115004 (2009).

2. Agterberg, D. F. et al. The physics of pair-density waves: cuprate superconductors and beyond. Annu. Rev. Condens. Matter Phys. 11, 231-270 (2020).

3. Fulde, P. \& Ferrell, R. A. Superconductivity in a strong spin-exchange field. Phys. Rev. 135, A550-A563 (1964).

4. Larkin, A. I. \& Ovchinnikov, Y. N. Nonuniform state of superconductors. Sov. Phys. JETP 20, 762-770 (1965).

5. Hamidian, M. H. et al. Detection of a cooper-pair density wave in $\mathrm{Bi}_{2} \mathrm{Sr}_{2} \mathrm{CaCu}_{2} \mathrm{O}_{8}$ $+x$. Nature 532, 343-347 (2016).

6. Ruan, W. et al. Visualization of the periodic modulation of cooper pairing in a cuprate superconductor. Nat. Phys. 14, 1178-1182 (2018).

7. Edkins, S. D. et al. Magnetic field-induced pair density wave state in the cuprate vortex halo. Science 364, 976-980 (2019).

8. Berg, E. et al. Dynamical layer decoupling in a stripe-ordered high- $\mathrm{T}_{c}$ superconductor. Phys. Rev. Lett. 99, 127003 (2007).

9. Agterberg, D. F. \& Tsunetsugu, H. Dislocations and vortices in pair-density-wave superconductors. Nat. Phys. 4, 639-642 (2008).

10. Jian, S.-K., Scherer, M. M. \& Yao, H. Mass hierarchy in collective modes of pairdensity-wave superconductors. Phys. Rev. Res. 2, 013034 (2020).

11. Berg, E., Fradkin, E. \& Kivelson, S. A. Pair-density-wave correlations in the kondoheisenberg model. Phys. Rev. Lett. 105, 146403 (2010).

12. Jaefari, A. \& Fradkin, E. Pair-density-wave superconducting order in two-leg ladders. Phys. Rev. B 85, 035104 (2012).

13. Han, Z., Kivelson, S. A. \& Yao, H. Strong coupling limit of the Holstein-Hubbard model. Phys. Rev. Lett. 125, 167001 (2020).

14. Huang, K. S., Han, Z., Kivelson, S. A. \& Yao, H. Pair-density-wave in the strong coupling limit of the Holstein-Hubbard model. Preprint at https://arxiv.org/abs/ 2103.04984 (2021).

15. Xu, X. Y., Law, K. T. \& Lee, P. A. Pair density wave in the doped $t-j$ model with ring exchange on a triangular lattice. Phys. Rev. Lett. 122, 167001 (2019).

16. Venderley, J. \& Kim, E.-A. Evidence of pair-density wave in spin-valley locked systems. Sci. Adv. 5, eaat4698 (2019).

17. Dodaro, J. F., Jiang, H.-C. \& Kivelson, S. A. Intertwined order in a frustrated four-leg $\mathrm{t}-\mathrm{J}$ cylinder. Phys. Rev. B 95, 155116 (2017).

18. Jiang, H.-C., Weng, Z.-Y. \& Kivelson, S. A. Superconductivity in the doped $\mathrm{t}-\mathrm{J}$ model: Results for four-leg cylinders. Phys. Rev. B 98, 140505 (2018).

19. Jiang, H.-C. \& Devereaux, T. P. Superconductivity in the doped Hubbard model and its interplay with next-nearest hopping t'. Science 365, 1424-1428 (2019). 
20. Jiang, Y.-F., Zaanen, J., Devereaux, T. P. \& Jiang, H.-C. Ground state phase diagram of the doped Hubbard model on the four-leg cylinder. Phys. Rev. Res. 2, 033073 (2020).

21. Balents, L. Spin liquids in frustrated magnets. Nature 464, 199-208 (2010).

22. Savary, L. \& Balents, L. Quantum spin liquids: a review. Rep. Prog. Phys. 80, 016502 (2016).

23. Broholm, C. et al. Quantum Spin Liquids. Science 367, eaay0668 (2020).

24. Anderson, P. W. The resonating valence bond state in $\mathrm{La}_{2} \mathrm{CuO}_{4}$ and superconductivity. Science 235, 1196-1198 (1987).

25. Kivelson, S. A., Rokhsar, D. S. \& Sethna, J. P. Topology of the resonating valence-bond state: solitons and high-T T $_{C}$ superconductivity. Phys. Rev. B 35, 8865-8868 (1987).

26. Rokhsar, D. S. \& Kivelson, S. A. Superconductivity and the quantum hard-core dimer gas. Phys. Rev. Lett. 61, 2376-2379 (1988).

27. Laughlin, R. B. The relationship between high-temperature superconductivity and the fractional quantum Hall effect. Science 242, 525-533 (1988).

28. Wen, X. G., Wilczek, F. \& Zee, A. Chiral spin states and superconductivity. Phys. Rev. B 39, 11413-11423 (1989).

29. Lee, P. A., Nagaosa, N. \& Wen, X.-G. Doping a mott insulator: physics of hightemperature superconductivity. Rev. Mod. Phys. 78, 17-85 (2006).

30. Lee, S.-S., Lee, P. A. \& Senthil, T. Amperean pairing instability in the U(1) spin liquid state with fermi surface and application to $\mathrm{K}-(\mathrm{BEDT}-\mathrm{TTF}) 2 \mathrm{Cu} 2(\mathrm{CN}) 3$. Phys. Rev. Lett. 98, 067006 (2007).

31. Fradkin, E., Kivelson, S. A. \& Tranquada, J. M. Colloquium: theory of intertwined orders in high temperature superconductors. Rev. Mod. Phys. 87, 457-482 (2015).

32. Jiang, H.-C. Superconductivity in the doped quantum spin liquid on the triangular lattice. Preprint at https://arxiv.org/abs/1912.06624 (2019).

33. Jiang, Y.-F. \& Jiang, H.-C. Topological superconductivity in the doped chiral spin liquid on the triangular lattice. Phys. Rev. Lett. 125, 157002 (2020).

34. Kitaev, A. Anyons in an exactly solved model and beyond. Ann. Phys. 321, 2- 111 (2006).

35. Rau, J. G., Lee, E. K.-H. \& Kee, H.-Y. Spin-orbit physics giving rise to novel phases in correlated systems: iridates and related materials. Annu. Rev. Condens. Matter Phys. 7, 195-221 (2016).

36. Mehlawat, K. \& Singh, Y. First-order density-wave-like transitions in surface-doped $\mathrm{Na}_{2} \mathrm{IrO}_{3}$. Phys. Rev. B 94, 041109 (2016).

37. Mehlawat, K. \& Singh, Y. Density wave like transport anomalies in surface doped $\mathrm{Na}_{2} \mathrm{IrO}_{3}$. AIP Adv. 7, 055710 (2017).

38. Banerjee, A. et al. Neutron scattering in the proximate quantum spin liquid $a$ $\mathrm{RuCl}_{3}$. Science 356, 1055-1059 (2017).

39. Takagi, H., Takayama, T., Jackeli, G., Khaliullin, G. \& Nagler, S. E. Concept and realization of kitaev quantum spin liquids. Nat. Rev. Phys. 1, 264-280 (2019).

40. Jiang, Y.-F., Devereaux, T. P. \& Jiang, H.-C. Field-induced quantum spin liquid in the kitaev-heisenberg model and its relation to $\alpha$-RuCl 3 . Phys. Rev. B 100, 165123 (2019).

41. You, Y.-Z., Kimchi, I. \& Vishwanath, A. Doping a spin-orbit mott insulator: Topological superconductivity from the kitaev-heisenberg model and possible application to $\mathrm{Na}_{2} / \mathrm{Li}_{2} \mathrm{IrO}$. Phys. Rev. B 86, 085145 (2012).

42. Hyart, T., Wright, A. R., Khaliullin, G. \& Rosenow, B. Competition between $d$-wave and topological $p$-wave superconducting phases in the doped kitaev-heisenberg model. Phys. Rev. B 85, 140510 (2012).

43. Okamoto, S. Global phase diagram of a doped kitaev-heisenberg model. Phys. Rev. B 87, 064508 (2013).

44. Schmidt, J., Scherer, D. D. \& Black-Schaffer, A. M. Topological superconductivity in the extended kitaev-heisenberg model. Phys. Rev. B 97, 014504 (2018).

45. Mei, J.-W. Possible fermi liquid in the lightly doped kitaev spin liquid. Phys. Rev. Lett. 108, 227207 (2012)

46. White, S. R. Density matrix formulation for quantum renormalization groups. Phys. Rev. Lett. 69, 2863-2866 (1992).

47. Jiang, H.-C., Devereaux, T. \& Kivelson, S. A. Holon wigner crystal in a lightly doped kagome quantum spin liquid. Phys. Rev. Lett. 119, 067002 (2017).

48. White, S. R., Affleck, I. \& Scalapino, D. J. Friedel oscillations and charge density waves in chains and ladders. Phys. Rev. B 65, 165122 (2002).

49. Moreno, A., Muramatsu, A. \& Manmana, S. R. Ground-state phase diagram of the one-dimensional $t$-j model. Phys. Rev. B 83, 205113 (2011).
50. Baskaran, G., Mandal, S. \& Shankar, R. Exact results for spin dynamics and fractionalization in the kitaev model. Phys. Rev. Lett. 98, 247201 (2007).

51. Calabrese, P. \& Cardy, J. Entanglement entropy and quantum field theory. J. Stat. Mech. Theory Exp. 2004, P06002 (2004).

52. Fagotti, M. \& Calabrese, P. Universal parity effects in the entanglement entropy of XX chains with open boundary conditions. J. Stat. Mech. Theory Exp. 2011, P01017 (2011).

53. Jiang, H. C., Wang, Z. \& Balents, L. Identifying topological order by entanglement entropy. Nat. Phys. 8, 902-905 (2012).

54. Zhu, Z., Kimchi, I., Sheng, D. N. \& Fu, L. Robust non-abelian spin liquid and a possible intermediate phase in the antiferromagnetic kitaev model with magnetic field. Phys. Rev. B 97, 241110 (2018).

\section{ACKNOWLEDGEMENTS}

We would like to thank Senthil Todadri and Edwin Huang for insightful discussions, and especially Steve Kivelson for insightful discussion, invaluable suggestions and generous help to understand the results and improve the manuscript. This work was supported by the Department of Energy, Office of Science, Basic Energy Sciences, Materials Sciences and Engineering Division, under Contract DE-AC02-76SF00515. Parts of the computing for this project was performed on the Sherlock cluster.

\section{AUTHOR CONTRIBUTIONS}

C.P. and Y.F.J. contributed equally. H.C.J. conceived the study. C.P., Y.F.J., and H.C.J. performed numerical simulations. C.P. analyzed data under the supervision of H.C.J. All authors assisted in data interpretation and contributed to writing the manuscript.

\section{COMPETING INTERESTS}

The authors declare no competing interests.

\section{ADDITIONAL INFORMATION}

Supplementary information The online version contains supplementary material available at https://doi.org/10.1038/s41535-021-00363-0.

Correspondence and requests for materials should be addressed to H.-C.J.

Reprints and permission information is available at http://www.nature.com/ reprints

Publisher's note Springer Nature remains neutral with regard to jurisdictional claims in published maps and institutional affiliations.

Open Access This article is licensed under a Creative Commons Attribution 4.0 International License, which permits use, sharing, adaptation, distribution and reproduction in any medium or format, as long as you give appropriate credit to the original author(s) and the source, provide a link to the Creative Commons license, and indicate if changes were made. The images or other third party material in this article are included in the article's Creative Commons license, unless indicated otherwise in a credit line to the material. If material is not included in the article's Creative Commons license and your intended use is not permitted by statutory regulation or exceeds the permitted use, you will need to obtain permission directly from the copyright holder. To view a copy of this license, visit http://creativecommons. org/licenses/by/4.0/.

(c) The Author(s) 2021 